\title{
Giant cell arteritis: immune and vascular aging as disease risk factors
}

\author{
Shalini V Mohan, Y Joyce Liao, Jonathan W Kim, Jörg J Goronzy and Cornelia M Weyand*
}

\begin{abstract}
Susceptibility for giant cell arteritis increases with chronological age, in parallel with age-related restructuring of the immune system and age-induced remodeling of the vascular wall. Immunosenescence results in shrinkage of the naïve T-cell pool, contraction of T-cell diversity, and impairment of innate immunity. Aging of immunocompetent cells forces the host to take alternative routes for protective immunity and confers risk for pathogenic immunity that causes chronic inflammatory tissue damage. Dwindling immunocompetence is particularly relevant as the aging host is forced to cope with an ever growing infectious load. Immunosenescence coincides with vascular aging during which the arterial wall undergoes dramatic structural changes and medium and large arteries lose their pliability and elasticity. On the molecular level, elastic fibers deteriorate and matrix proteins accumulate biochemical modifications. Thus, the aging process impacts the two major biologic systems that liaise to promote giant cell arteritis; the immune system and the vessel wall niche.
\end{abstract}

\section{Introduction}

Giant cell arteritis (GCA) is a granulomatous disease that displays tissue tropism for large and medium arteries manifesting as aortitis and vasculitis of the second to fifth branches of the aorta [1]. Granulomatous lesions are typically localized in the wall layers of the affected arteries; extra-vascular GCA is rare, and likely represents a distinct entity. The arteritis is almost always combined with intense systemic inflammation and a powerful acute phase response. Similar to other inflammatory syndromes,

*Correspondence: cweyand@stanford.edu

Department of Medicine, Division of Immunology and Rheumatology and the Department of Ophthalmology, Stanford University School of Medicine, Stanford, CA 94305-5166, USA
GCA is a complex disorder with multiple pathogenic factors. An instigator initiating the inflammatory process has not been identified; however, overwhelming evidence has accumulated that abnormalities in innate and adaptive immunity play a critical role in the initiation and the perpetuation of the vasculitis.

Several unique factors of GCA have been informative in dissecting its immunopathogenesis. The disease is characterized by a stringent tissue tropism; meaning that granulomatous wall infiltrates typically appear in arteries of selected vascular beds. This pathogenic feature strongly suggests that vessel-wall-specific factors drive GCA. Dendritic cells (DCs), similar to skin-residing Langerhans cells, have been implicated in providing initial signals that break the immune protection of arterial walls $[2,3]$. Vascular DCs are an endogenous cell population in the arteries. They define the immunological identity of blood vessels by expressing a vessel-specific profile of pattern recognition receptors [4]. Activated vascular DCs recruit adaptive immune responses, sustain them in the vascular wall and shape their architecture and functional direction. The nature of the adaptive immune response underlying the granulomatous reaction is now well understood [5]. In essence, a selected population of $\mathrm{T}$ lymphocytes travels to the arterial lesion, undergoes local proliferation and activation and produces pro-inflammatory cytokines regulating the functions of macrophages, vascular smooth muscle cells and endothelial cells. T cells that accumulate in the granulomatous lesions are non-random; identical $\mathrm{T}$ cells have been isolated from the right and left temporal artery of the same patient [6]. The selectivity of the T cells involved in the disease process is highly suggestive for antigen as the ultimate driver of the inflammation. Vasculitis T cells are functionally selected as well, and belong to either the Thelper (Th) 1 or the Th17 lineage [7]. In GCA patients, the two T-cell lineages are differentially responsive to corticosteroid therapy and appear to be responsible for distinct aspects of the vasculitis process [8]. The multiplicity of T-cell abnormalities suggests complexity in disease-driving signals, emphasizes the intricacy of the immune-mediated damage to the blood vessel and encourages multifaceted therapeutic approaches. 
While substantial progress has been made in dissecting the T-cell biology of GCA, one critical disease factor has been less well examined. The single strongest risk factor is age, with incidence steadily rising in the seventh to eighth decades of life $[9,10]$. The disease essentially does not exist in individuals younger than 50 years of age. Progressive age could pose a risk to develop GCA through two major trajectories: age-related remodeling of the immune system, both the innate as well as the adaptive arm; and aging-imposed restructuring of the blood vessel wall. Takayasu's arteritis, a granulomatous vasculitis of the aorta and its primary branches, affects individuals prior to age 40 years and thus unfolds on the background of a distinct immune system and a distinct vascular microenvironment. Understanding the impact of the aging process on the immune system and the vascular system are necessary components of a more comprehensive concept of GCA, its risk factors and its manageable pathogenic elements.

\section{Age and the immune system}

The human immune system is designed to protect the host from infections, destroy malignant cells, regulate tissue healing and prevent immune responses to self. Over a lifetime, the immune system is challenged with a broad spectrum of infectious pathogens and responds with massive expansion of the cells required to destroy the invader and memorize the encounter for future protection. The constant need to protect the host thus imposes enormous proliferative stress upon the bonemarrow-derived cells that differentiate into immunecompetent cell types. With progressive age, production of new immune cells deteriorates. This is particularly relevant for $\mathrm{T}$ lymphocytes as involution of the thymus essentially abrogates regeneration of $\mathrm{T}$ cells in middle age. The question arises whether age-imposed changes in the pool of $\mathrm{T}$ lymphocytes increases the risk for GCA.

The term immunosenescence is now used for a series of changes that are typically encountered in the immune system of the elderly (Table 1) [11]. First and foremost, the production of naïve $\mathrm{T}$ cells declines. In the thymus, bone-marrow-derived precursor cells develop into mature $\mathrm{T}$ cells and are checked for autoreactivity, at which time auto-reactive $\mathrm{T}$ cells are eliminated. $\mathrm{T}$ cells with low-affinity for self antigens are released into the periphery where they form the pool of naïve $\mathrm{T}$ cells. Once $\mathrm{T}$ cells encounter their specific antigen, they enter a differentiation process and turn into memory and effector T cells. During middle age, the process of thymic $\mathrm{T}$-cell production declines markedly and the naïve T-cell pool shrinks. Within the T-cell compartment, enddifferentiated memory and effector $\mathrm{T}$ cells replace naïve $\mathrm{T}$ cells. Chronic persistent infections place a particular strain on the immune system. Viral persistence, such as

\section{Table 1. The signature of immunosenescence}

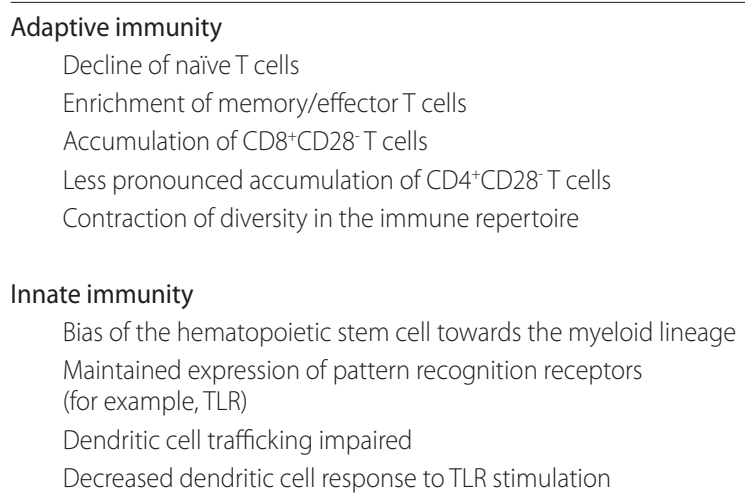

Environmental stressors

Increased infectious load

Reactivation of persistent infections

TLR, Toll-like receptor.

in cytomegalovirus infection, can drive chronic immune stimulation, which gradually exhausts the reserve of naïve $\mathrm{T}$ cells [12].

A prototypic change in end-differentiated $\mathrm{T}$ cells is the loss of the CD28 receptor from the cell surface. $\mathrm{CD} 4{ }^{+} \mathrm{CD} 28^{-} \mathrm{T}$ cells were first observed in patients with rheumatoid arthritis (RA) and their accumulation and clonality has given rise to the concept that RA patients have premature immune aging [13]. Other abnormalities typically encountered in the old are shifts in the distribution of CD4 and CD8 cells, a contraction in T-cell receptor diversity and loss of T-cell telomeres [14]. From studies testing vaccine responses in the elderly, it is known that only a fraction of over-65-year olds are capable of mounting protective immune responses and that the frequency of senescent $\mathrm{CD} 8^{+} \mathrm{CD} 28^{-} \mathrm{T}$ cells is an excellent predictor of non-responsiveness [15].

It is easy to understand that progressive age and contracting numbers of naïve $\mathrm{T}$ cells go hand in hand with declining immunity. It is more difficult to understand how the derangements of the T-cell system affect the ability to tolerate self. It is now clear that selftolerance is an active mechanism that depends on a wellregulated immune system. The strongest evidence that the aging immune system loses the capability to fine-tune immune responses and suppress those that would lead to self damage comes from the observation that the very old often have a state of chronic smoldering inflammation [16]. This chronic, low-grade inflammation is sometimes called inflamm-aging. Cytokines, such as IL-6, TNF- $\alpha$, and IL-1 $\beta$, are suspected to have an important role in sustaining chronic inflammation (Figure 1). Such cytokines mainly derive from the innate immune system, suggesting that immunosenescence affects both the innate and adaptive immune systems. Continuous immune 


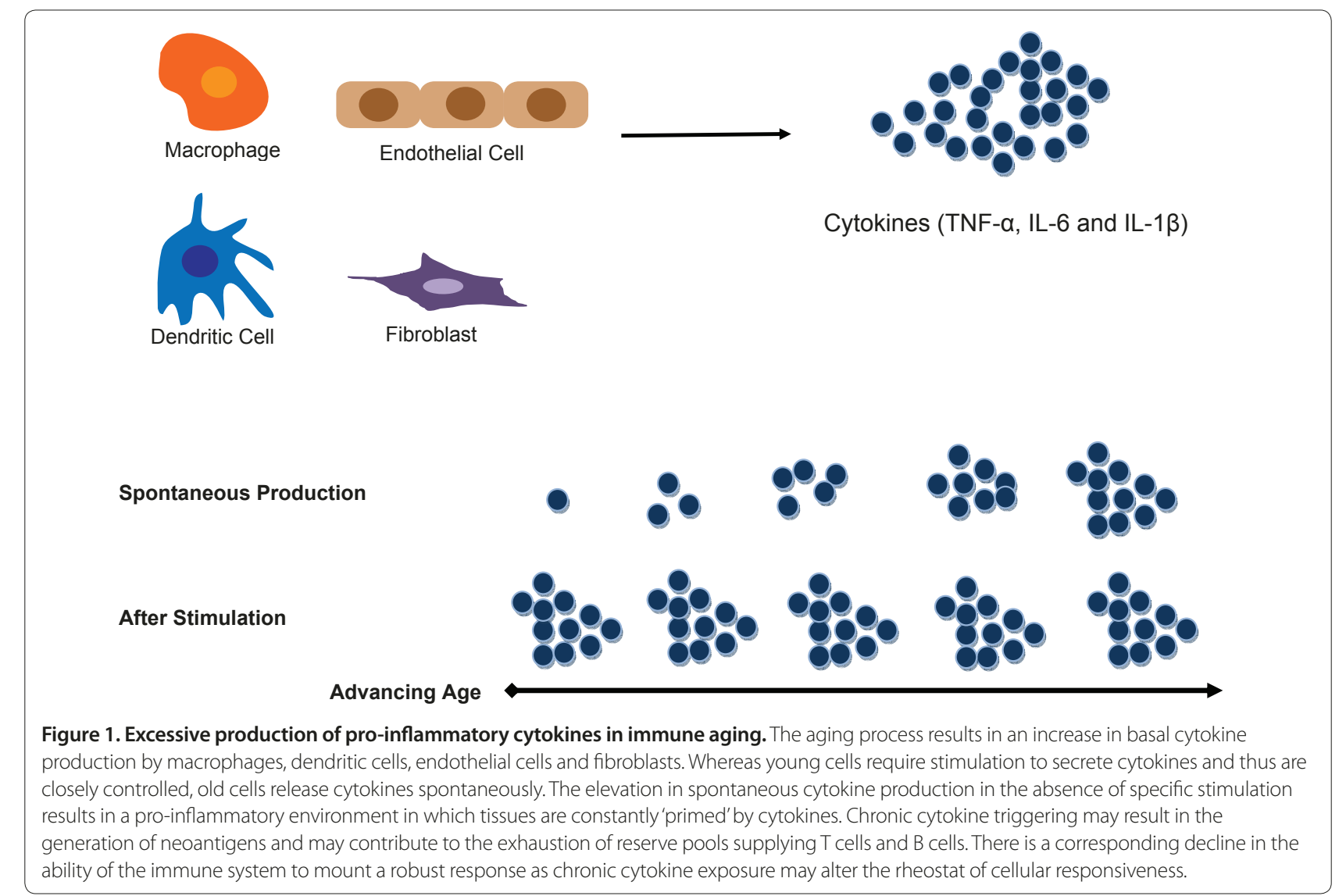

stimulation, as well as release of pro-inflammatory cytokines from other senescent cells, such as fibroblasts, have also been implicated as causative factors. Thus, ageinduced abnormalities in immune and non-immune cells may join forces to promote inflamm-aging (Figure 1). Parallel changes in the tissue microenvironment, leaving tissue-residing cells with a primed phenotype, could easily generate tissues that are more susceptible to the action of systemically produced pro-inflammatory cytokines. The end result would be that enhanced synthesis of pro-inflammatory mediators, such as IL- 6 , TNF- $\alpha$, and IL-1 $\beta$, exacerbates relatively benign tissue inflammation and switches it into a destructive event.

In summary, immunosenescence describes a series of abnormalities in the innate and adaptive immune system (Table 1) that create a state of chronic inflammation, impair anti-pathogen immune responses, increase susceptibility to malignancy and autoimmunity, decrease anti-vaccine responses and undermine wound healing. It is conceivable that such abnormalities also render individuals susceptible to vessel wall inflammation.

\section{Aging of $T$ cells and dendritic cells}

The central cellular players in GCA are vessel-embedded DCs that sense danger signals and $\mathrm{T}$ cells that mount an adaptive immune response at the tissue site [17]. Both cell types are subject to aging, directly connecting immunosenescence and GCA susceptibility. T-cell aging is mostly determined by the inability of the thymus to replenish new $\mathrm{T}$ cells while maintaining memory $\mathrm{T}$-cell responses and keeping check of chronic persistent infections [18]. Interestingly, aging of CD4 and CD8 $\mathrm{T}$ cells proceeds at different speeds [14]. CD8 T cells age faster, accumulate more $\mathrm{CD} 28^{-}$cells and display a higher degree of clonality. This may be a consequence of persistent viral infections, such as cytomegalovirus, which impose considerable stress upon CD8 T cells and induce restructuring of the immune system with progressive age. Little is known about the role of CD8 $T$ cells in GCA, other than the fact that they account for only a minor part of the vasculitic infiltrate. Reports in the 1990s drew attention to the blood CD8 compartment in GCA $[19,20]$.

A study performed in Denmark with 227 active, untreated GCA patients and 227 age- and gendermatched controls revealed a significant reduction in the CD8 population compared to controls (12\% CD8 cells in patients versus $20 \%$ in age-matched controls). In several subsequent studies, decreased CD8 T-cell counts could not be confirmed as a universal marker of GCA. Rather, 
analysis of T-cell clonality revealed that the CD4/CD8 ratio in both patients and controls correlated closely with the number of clonal T-cell populations [21,22].

$\mathrm{CD} 4 \mathrm{~T}$ cells, the major component of the GCA wall lesions, age slower, but essentially undergo similar changes as CD8 T cells with progressive age [14]. Detailed studies have shown that the aging process in CD4 cells is significantly accelerated in RA, an autoimmune syndrome with chronic inflammation of the synovial membrane and more rapid atherosclerosis. RA has emerged as a model system to study the interrelationship between chronic inflammation and immune aging. Molecular defects described in RA patients may provide helpful insights into how the immune system ages overall and which particular pathways are relevant in the setting of chronic inflammation. In RA patients, CD4 T-cell immunosenescence has been molecularly linked to a defect in repairing damaged DNA. This defect involves the telomeric ends as well as non-telomeric genomic DNA. $\mathrm{T}$ cells from RA patients fail to properly upregulate telomerase and thus are much less efficient in protecting and repairing telomeres [23]. Also, $\mathrm{T}$ cells from RA patients have impaired upregulation of ataxia telangiectasia mutated (ATM), a kinase critically relevant in recognizing double-strand breaks of non-telomeric DNA $[16,24]$. ATM has been implicated in participating in maintaining genomic integrity of the telomeric ends as well. In $\mathrm{T}$ cells from RA patients, damaged DNA accumulates and DNA repair mechanisms are blunted. Forced overexpression of ATM restores the ability to repair fragmented DNA and extends the life span of RA $T$ cells. RA T cells sense that they fail to fix DNA damage and chronically activate cell internal stress pathways $[24,25]$. It is currently unknown how these molecular networks are regulated in patients who have GCA. However, systemic inflammation may undermine DNA damage recognition and repair, and therefore accelerate aging of the T-cell compartment. Recognizing the role of the DNA repair machinery in determining immunosenescence provides access to possible interventions to counteract the aging process in the immune system (Table 2). T cells orchestrate immune responses, both protective as well as harmful, by releasing cytokines. Recently, two cytokine-committed T-cell lineages have been implicated in GCA [7]. Th1 cells produce IFN- $\gamma$, target macrophages and provide strong pro-inflammatory function. In earlier studies, tissue IFN- $\gamma$ levels have been correlated with the production of vascular endothelial growth factor and platelet-derived growth factor, molecules implicated in the neointimal response that leads to luminal stenosis [26,27]. Th17 cells secrete IL-17, a cytokine that is known to regulate recruitment of neutrophils and provide protective immunity during early immune reactions, particularly indolent infections.

\section{Table 2. Molecules involved in premature immune aging}

Telomerase

Insufficient repair of telomeric ends in old T cells

Ataxia telangiectasia mutated

Blunted repair of DNA double-strand breaks in old T cells

DNA-dependent protein kinase catalytic subunit (DNA-PKCS)

Excessive activation of the DNA repair machinery with triggering of cellular stress pathways in old T cells

Reactive oxygen species

Impaired cellular detoxification of reactive oxygen species, accumulation of oxidized lipids and proteins, modification of signaling proteins and membranes

Both Th1 and Th17 cells participate in GCA lesions. Th17 cells can be relatively easily suppressed with corticosteroids, whereas Th1 cells are steroid-resistant and persist into the chronic phase of disease. Recent studies have refined the characterization of vasculitic $\mathrm{T}$ cells and have demonstrated that expression of the $\mathrm{NOTCH} 1$ receptor on $\mathrm{T}$ cells has an immediate role in the inflammatory process [28].

Th17 cells have also been implicated in atherosclerosis, a prototypic age-related syndrome in which mechanisms of immune and vascular aging interplay. Cells producing IFN- $\gamma$ and IL-17 have been found in atherosclerotic plaques and are thought to create a pro-inflammatory environment that causes abnormal function of vascular smooth muscle cells that eventually leads to plaque growth and instability [29]. It is possible that the aged immune system is biased towards the generation of Th1 cells, instead of relying more on Th17 cells.

Whether DCs age at their own pace or in tandem with $\mathrm{T}$ cells, their major partner in the immune system, is yet unresolved. DC functions amenable to the aging process include their production, their homing to peripheral tissues, their responsiveness to 'danger signals', their migration from the periphery to central lymphoid organs and their direct interaction with $\mathrm{T}$ cells in the priming process. Studies of DC populations in GCA lesions have revealed that they are the critical antigen-presenting cells during early and late stages of disease [2]. They display an unusual phenotype in that they express the CCR7 chemokine receptor. CCR7 expression on DCs is a sign of maturation and enables the DCs to follow a gradient of CCL19 and CCL21, two chemokines that direct the cells through lymph vessels into lymph nodes. In GCA lesions, DCs themselves produce high amounts of CCL19 and CCL21, triggering local trapping of the cells and preventing them from leaving the inflammatory tissue niche [30].

Two studies in the human system and several studies in murine systems have approached the question of whether 
DC function changes with aging and how this affects immune-competence. In an influenza vaccine response study, Toll-like receptor (TLR) function of human DCs was assessed among 104 subjects. Older individuals were found to have DCs with lower TNF- $\alpha$ and IFN- $\alpha$ production in response to TLR stimulation compared to younger subjects. Older individuals also had higher intracellular cytokine levels in the absence of TLR triggering compared to young [31]. In the murine model, young and old marrow-derived DCs were compared, and it has been shown that in vivo DC migration and CCR7 signal transduction are defective in aged DCs compared to their younger counterparts. Though the migratory defect could be overcome by increasing the numbers of DCs, the defect in CD8 T-cell priming persisted [32]. The findings of these studies support the idea that DC aging impairs functional activity and that they may contribute to the observed inflamm-aging seen in the elderly. Other studies in both human and mice have shown that DCs, particularly plasmacytoid DCs, are susceptible to the aging process and this occurs both via decrease in population size and TLR function [33-36]. A molecular profile of the age-related changes in T-cell and DC function that renders individuals susceptible to GCA or is caused by the inflammatory activity in GCA has yet to be defined.

Vascular aging as a risk factor for giant cell arteritis GCA displays a stringent tissue tropism for certain vascular territories. Such tissue tropism may result from selective availability of disease-relevant antigens. Alternatively, vessel wall cell populations may contribute to disease initiation and the composition of the vessel wall may not be homogeneous through the vascular tree. Studies performed over the past 5 years have lent strong support for the second alternative. Specifically, a population of immune-sensing cells, vascular DCs, have been identified in the walls of medium and large arteries [17]. A population of vascular DCs is positioned at the mediaadventitia border of the human aorta and carotids, as well as subclavian, mesenteric, iliac and temporal arteries. Such vascular DCs provide immunosurveillance functions by sensing pathogen-derived molecular patterns (PAMPs). They utilize a spectrum of TLRs for this sensing function [4]. Remarkably, studies of normal human arteries from distinct vascular beds have shown that the TLR profile in each arterial territory is vesselspecific and unique [4]. Thus, human arteries contribute to monitoring immune danger and they do so in a very selective way. Granulomatous lesions contain highly activated DCs, which have been implicated in promoting activation of vasculitic $\mathrm{T}$ cells [2]. Atherosclerotic plaques, another type of vascular lesion, are also populated by DCs, which shape the inflammatory milieu and activate $\mathrm{T}$ cells [37]. Whether such lesional DCs derive directly from the wall-residing DC population or whether they are recruited from the blood stream is currently not known.

Vascular DCs have been implicated in initiating and sustaining mural inflammation. Signals received by vascular DCs determine the microarchitecture of the evolving vascular inflammation [38] and DCs are indispensable for the recruitment and the guidance of $\mathrm{T}$ cells that enter the vessel wall [3]. Studies in human artery severe combined immunodeficiency (SCID) mouse chimeras have demonstrated that each artery displays a selective sensing function and that each artery interacts with $\mathrm{T}$ cells in a vessel-specific pattern. In essence, at least part of the tissue tropism for GCA results from vessel-specific immune functions. Immune-sensing functions of blood vessels may well be subject to age-related changes. It is not yet fully understood how the number, distribution, mobility and responsiveness to danger signals changes in vascular DCs as the host ages. However, substantial changes in the wall structure make it almost inevitable that vascular DCs in an 80-year old function distinctly from those in a 20-year old.

Functionality of the TLR-based sensing machinery in human dendritic cells is definitely susceptible to agerelated changes. Both myeloid and plasmacytoid DCs have been shown to be less responsive to TLR stimulation in older individuals [32]. While a decline in TLR responsiveness may suggest less pro-inflammatory functions deriving from 'old' DCs, such 'old' DCs would also loose anti-inflammatory functions. Tolerogenicity of DCs is equally dependent on proper sensing functions. Deterioration of tolerogenecity with advancing age would thus turn tolerogenic DCs into immune stimulator DCs (Figure 2). The relative abundance of systemically distributed pro-inflammatory cytokines (for example, IL-6, TNF- $\alpha$, IL-1) may indeed alter DC behavior and bias DCs towards a different maturation program. It is well established that older individuals fail to generate protective immune responses with similar efficiency as younger counterparts [15,39]. While declining T-cell immunity has a central role in immunosenescence, DCs remain the major partners for $\mathrm{T}$ cells and are equally important in initiating and sustaining immune responses. Functional consequences for vascular DCs when chronically exposed to a milieu of inflammation need to be explored.

Age-induced changes in blood vessel structure and function are not limited to wall-embedded immunecompetent DCs. From macroscopic and microscopic studies, it has long been known that the medial layer is prone to age-related degeneration [40,41] (Figure 3). Vascular smooth muscle cells decrease in number and function. The media becomes thinner and deposition of calcium is not unusual. Overall, the arterial wall can 


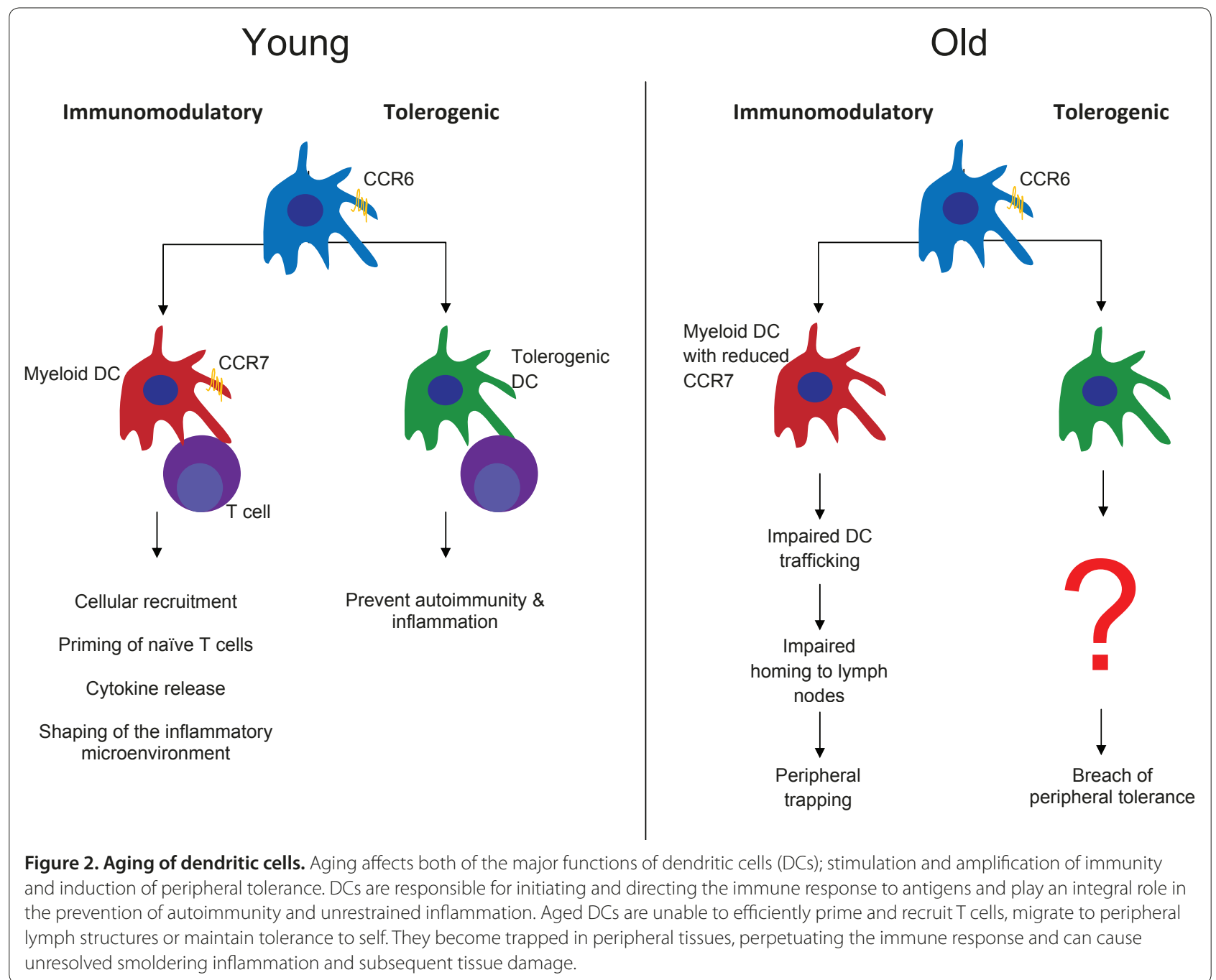

thicken because the intimal layer expands from one to several layers. Elasticity of the arteries, mediated by the elastic fibers and membranes integrated into the media, deteriorates, clinically recognized as increased stiffness [42]. The underlying mechanism relates to the fracture of elastic membranes and the fizzing of elastic fibers [43]. As life progresses, matrix proteins responsible for the facilitation of cell-cell interactions and the transmission of forces throughout the wall structure are biochemically modified; changing structural features and transmural trafficking patterns for cells and molecules. Matrix proteins also serve as reservoirs for molecules released from wall-residing cells that promote communication between different layers. In essence, the tissue niche of the vessel wall is profoundly altered by the aging process. Biochemical changes associated with vascular aging may actually be accelerated by inflammatory damage of wall structures. In particular, vasculitis typically leads to fragmentation of elastic membranes and destruction of cell-protective matrix layers.
One of the molecular pathways implicated in molecular aging relates to oxidative stress, a process incriminated in aging overall [44]. As humans age, protective mechanisms detoxifying oxygen radicals are impaired. As a consequence, reactive oxygen species are more plentiful in the aged host. Such highly reactive oxygen species, derivatives of metabolic processes in the cell, are able to induce multiple biochemical modifications of proteins as well as membrane-integrated lipids. Aging of the aorta is associated with accumulation of advanced glycation endproducts and Alcian-blue positive mucin within the medial layer [41]. Glycation end-products have so far not been reported in medium-sized arteries, such as the temporal artery. Finally, endothelial cells are subject to aging. As prototypic vascular components, they not only line the macrolumen of medium and large arteries, but also form the microvascular vasa vasorum tree. Many of the immune recognition processes involved in GCA are located in the adventitia and the adventitia-adjacent media [45]. This is the neighborhood dominated by the 


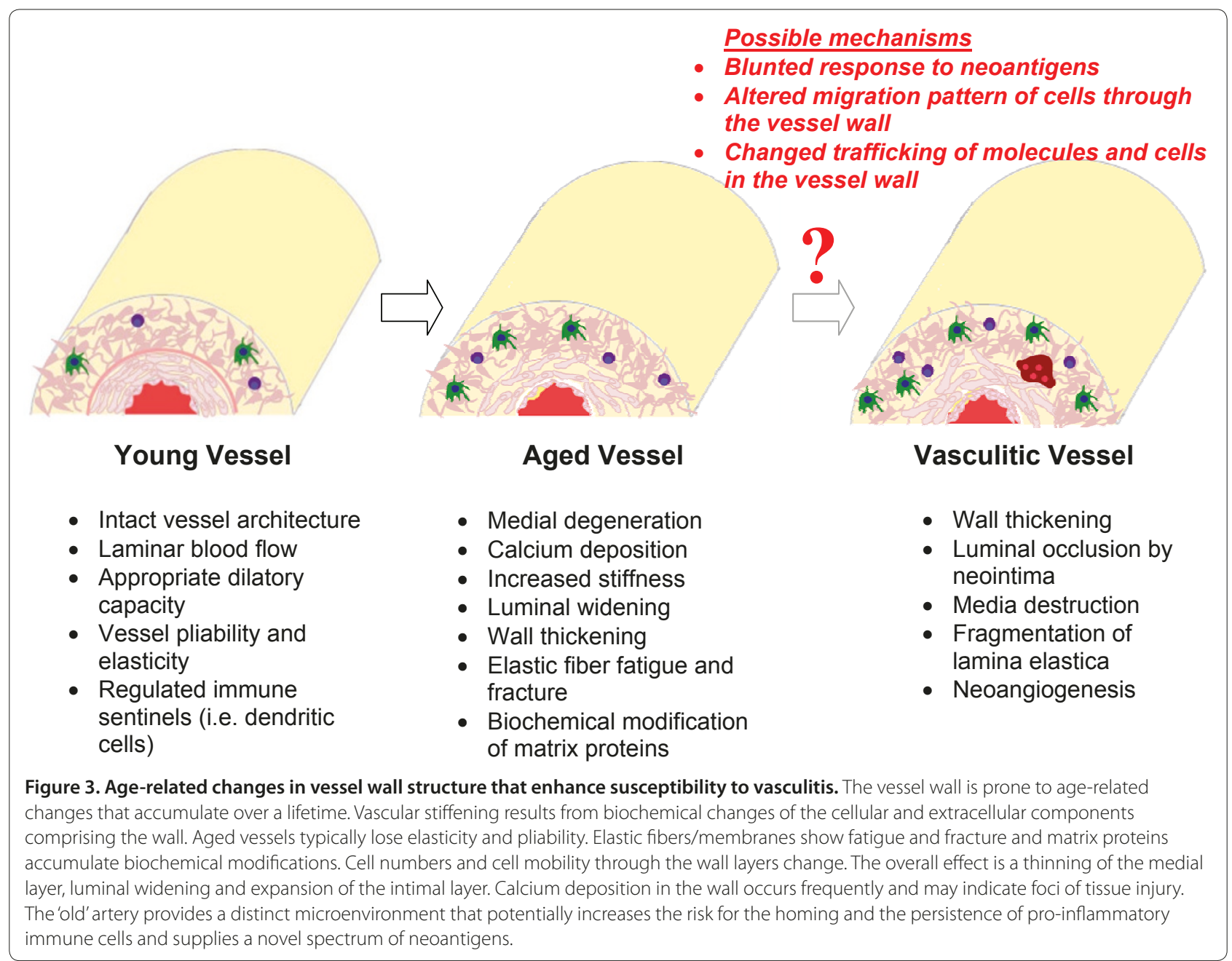

vasa vasorum network. Disorganization of the vasa vasorum tree has been identified as an early abnormality in atherosclerotic disease [46]. Vasa vasora provide the entrance for inflammatory cells in GCA lesions. Whether they have additional immunoregulatory functions in the early and late steps of the vasculitis is currently unknown. Insufficient regeneration of endothelial layers by endothelial precursor cells has been suspected as an important disease mechanism in multiple vascular pathologies. Organ-specific precursor cell populations decline with advancing age. Here, the aging process would have profound consequences for vessel wall integrity and function.

\section{Conclusion}

Progressive age, the strongest of all risk factors for GCA, impacts the two major elements that interact to cause vascular inflammation; the innate and adaptive arms of the immune system and the unique microenvironment of the vessel wall. Clinically, immune aging is characterized by the loss of protective immunity and the occurrence of host-injurious inflammation. Failure to protect the host results from the decline of immune cell regeneration. As a consequence, the elderly accumulate an increasing load of pathogens. Cells that survive in the aging immune system are less well controlled, spontaneously release cytokines and have altered threshold settings in response to immune stimuli. GCA is a disease of uncontrolled DC and $\mathrm{T}$-cell activation in a tissue site that is ordinarily inaccessible for immune responses. As the host ages, the tissue site itself undergoes numerous structural changes, summarized as vascular aging. This process brings with it changes in elastic fibers, matrix proteins and remodeling of the medial and intimal layer, affecting cellular traffic, turnover and survival. An attractive hypothesis is that the infectious load of the aging host triggers activation of vascular DCs that can no longer protect the structurally altered vascular wall, giving rise to tissue-injurious inflammation. Studies are needed to precisely define how the aging process affects GCA-susceptible blood vessels and how vascular DCs change functionally and molecularly as the host ages. 
Recent work has identified at least two lineages of proinflammatory T cells contributing to GCA; Th1 and Th17 cells. It is currently unknown whether the process of immunosenescence affects Th1 and Th17 cells in a lineage-specific way or whether both T-cell types age in parallel. The molecular details of T-cell aging in GCA remain to be elucidated. In GCA patients, Th1 and Th17 cells differ in therapeutic responsiveness and participation in early and chronic disease. Evidence suggests that the two lineages respond to distinct instigators. A combination of pathogen-derived and tissue-indigenous danger signals may result in the chronic granulomatous inflammation that characterizes GCA. Therapeutic approaches for GCA patients need to be tailored to the special needs of the aging host. Immunosuppression needs to be restricted to avoid further weakening of immunocompetence. DCs originate from the bone marrow and the development of DC-target therapies will require a much better understanding of their life cycle. Enhancing immune cell regeneration could help in clearing chronic infections. Prevention of vascular aging is a long-drawn process that should begin during middle age. Given similarities in the inflammatory process that typifies atherosclerosis and GCA, it is possible that managing standard vascular risk factors could function as adjuvant therapy in the treatment of large vessel vasculitis.

Autoimmune Basis of Rheumatic Diseases

This article is part of a review series on Vasculitis, edited by Cees

Kallenberg, which can be found online at

http://arthritis-research.com/series/vasculitis

This series forms part of a special collection of reviews covering major autoimmune rheumatic diseases, available at:

http://arthritis-research.com/series/abrd

\section{Abbreviations}

ATM, ataxia telangiectasia mutated; DC, dendritic cell; GCA, giant cell arteritis; IFN, interferon; IL, interleukin; RA, rheumatoid arthritis; Th, T-helper; TLR, Tolllike receptor; TNF, tumor necrosis factor.

\section{Competing interests}

The authors declare that they have no competing interests.

Published: 2 August 2011

\section{References}

1. Weyand CM, Goronzy JJ: Medium- and large-vessel vasculitis. N Engl J Med 2003, 349:160-169.

2. Ma-Krupa W, Jeon MS, Spoerl S, Tedder TF, Goronzy JJ, Weyand CM: Activation of arterial wall dendritic cells and breakdown of self-tolerance in giant cell arteritis. J Exp Med 2004, 199:173-183.

3. Han JW, Shimada K, Ma-Krupa W, Johnson TL, Nerem RM, Goronzy JJ, Weyand CM: Vessel wall-embedded dendritic cells induce T-cell autoreactivity and initiate vascular inflammation. Circ Res 2008, 102:546-553.

4. Pryshchep O, Ma-Krupa W, Younge BR, Goronzy JJ, Weyand CM: Vesselspecific Toll-like receptor profiles in human medium and large arteries. Circulation 2008, 118:1276-1284.
5. Weyand CM, Younge BR, Goronzy JJ: T cells in arteritis and atherosclerosis. Curr Opin Lipido/ 2008, 19:469-477.

6. Weyand CM, Schonberger J, Oppitz U, Hunder NN, Hicok KC, Goronzy JJ: Distinct vascular lesions in giant cell arteritis share identical T cell clonotypes. J Exp Med 1994, 179:951-960.

7. Weyand CM, Younge BR, Goronzy JJ: IFN-gamma and IL-17: the two faces of T-cell pathology in giant cell arteritis. Curr Opin Rheumatol 2011, 23:43-49.

8. Deng J, Younge BR, Olshen RA, Goronzy JJ, Weyand CM: Th17 and Th1 T-cell responses in giant cell arteritis. Circulation 2010, 121:906-915.

9. Nordborg E, Nordborg C: Giant cell arteritis: epidemiological clues to its pathogenesis and an update on its treatment. Rheumatology (Oxford) 2003 42:413-421.

10. Weyand CM, Goronzy JJ: Giant-cell arteritis and polymyalgia rheumatica. Ann Intern Med 2003, 139:505-515.

11. Goronzy JJ, Lee WW, Weyand CM: Aging and T-cell diversity. Exp Gerontol 2007, 42:400-406.

12. Moss $P$ : The emerging role of cytomegalovirus in driving immune senescence: a novel therapeutic opportunity for improving health in the elderly. Curr Opin Immunol 2010, 22:529-534.

13. Weyand CM, Fujii H, Shao L, Goronzy JJ: Rejuvenating the immune system in rheumatoid arthritis. Nat Rev Rheumato/ 2009, 5:583-588.

14. Czesnikiewicz-Guzik M, Lee WW, Cui D, Hiruma Y, Lamar DL, Yang ZZ, Ouslander JG, Weyand CM, Goronzy JJ: T cell subset-specific susceptibility to aging. Clin Immuno/ 2008, 127:107-118.

15. Goronzy JJ, Fulbright JW, Crowson CS, Poland GA, O'Fallon WM, Weyand CM: Value of immunological markers in predicting responsiveness to influenza vaccination in elderly individuals. J Viro/ 2001, 75:12182-12187.

16. Campisi J, d'Adda di Fagagna F: Cellular senescence: when bad things happen to good cells. Nat Rev Mol Cell Biol 2007, 8:729-740

17. Weyand CM, Ma-Krupa W, Pryshchep O, Groschel S, Bernardino R, Goronzy JJ: Vascular dendritic cells in giant cell arteritis. Ann N Y Acad Sci 2005, 1062:195-208.

18. Vallejo AN, Weyand CM, Goronzy JJ: T-cell senescence: a culprit of immune abnormalities in chronic inflammation and persistent infection. Trends $\mathrm{Mol}$ Med 2004, 10:119-124.

19. Arnold MH, Corrigall VM, Pitzalis C, Panayi GS: The sensitivity and specificity of reduced CD8 lymphocyte levels in the diagnosis of polymyalgia rheumatica/giant cell arteritis. Clin Exp Rheumatol 1993, 11:629-634.

20. Elling $\mathrm{P}, \mathrm{Olsson} A \mathrm{AT}$, Elling $\mathrm{H}$ : A reduced CD8+ lymphocyte subset distinguishes patients with polymyalgia rheumatica and temporal arteritis from patients with other diseases. Clin Exp Rheumatol 1998, 16:155-160.

21. Martinez-Taboada VM, Goronzy JJ, Weyand CM: Clonally expanded CD8 T cells in patients with polymyalgia rheumatica and giant cell arteritis. Clin Immunol Immunopathol 1996, 79:263-270.

22. Martinez-Taboada VM, Blanco R, Fito C, Pacheco MJ, Delgado-Rodriguez M, Rodriguez-Valverde V: Circulating CD8+ T cells in polymyalgia rheumatica and giant cell arteritis: a review. Semin Arthritis Rheum 2001, 30:257-271.

23. Fujii H, Shao L, Colmegna I, Goronzy JJ, Weyand CM: Telomerase insufficiency in rheumatoid arthritis. Proc Natl Acad Sci U S A 2009, 106:4360-4365.

24. Shao L, Fujii H, Colmegna I, Oishi H, Goronzy JJ, Weyand CM: Deficiency of the DNA repair enzyme ATM in rheumatoid arthritis. J Exp Med 2009, 206:1435-1449.

25. Shao L, Goronzy JJ, Weyand CM: DNA-dependent protein kinase catalytic subunit mediates T-cell loss in rheumatoid arthritis. EMBO Mol Med 2010, 2:415-427.

26. Kaiser M, Younge B, Bjornsson J, Goronzy JJ, Weyand CM: Formation of new vasa vasorum in vasculitis. Production of angiogenic cytokines by multinucleated giant cells. Am J Pathol 1999, 155:765-774

27. Kaiser M, Weyand CM, Bjornsson J, Goronzy JJ: Platelet-derived growth factor, intimal hyperplasia, and ischemic complications in giant cell arteritis. Arthritis Rheum 1998, 41:623-633.

28. Piggott K, Deng J, Warrington K, Younge B, Kubo JT, Desai M, Goronzy JJ, Weyand CM: Blocking the NOTCH pathway inhibits vascular inflammation in large-vessel vasculitis. Circulation 2011, 123:309-318.

29. Eid RE, Rao DA, Zhou J, Lo SF, Ranjbaran H, Gallo A, Sokol SI, Pfau S, Pober JS, Tellides G: Interleukin-17 and interferon-gamma are produced concomitantly by human coronary artery-infiltrating $T$ cells and act synergistically on vascular smooth muscle cells. Circulation 2009, 119:1424-1432.

30. Krupa WM, Dewan M, Jeon MS, Kurtin PJ, Younge BR, Goronzy JJ, Weyand CM: 
Trapping of misdirected dendritic cells in the granulomatous lesions of giant cell arteritis. Am J Pathol 2002, 161:1815-1823.

31. Panda A, Qian F, Mohanty S, van Duin D, Newman FK, Zhang L, Chen S, Towle V, Belshe RB, Fikrig E, Allore HG, Montgomery RR, Shaw AC: Age-associated decrease in TLR function in primary human dendritic cells predicts influenza vaccine response. J Immunol 2010, 184:2518-2527.

32. Grolleau-Julius A, Harning EK, Abernathy LM, Yung RL: Impaired dendritic cell function in aging leads to defective antitumor immunity. Cancer Res 2008, 68:6341-6349

33. Grolleau-Julius A, Garg MR, Mo R, Stoolman LL, Yung RL: Effect of aging on bone marrow-derived murine $\mathrm{CD} 11 \mathrm{c}+\mathrm{CD} 4-\mathrm{CD} 8 \mathrm{alpha}$ - dendritic cell function. J Gerontol A Biol Sci Med Sci 2006, 61:1039-1047.

34. Tesar BM, Walker WE, Unternaehrer J, Joshi NS, Chandele A, Haynes L, Kaech S, Goldstein DR: Murine [corrected] myeloid dendritic cell-dependent tolllike receptor immunity is preserved with aging. Aging Cell 2006, 5:473-486

35. Stout-Delgado HW, Yang X, Walker WE, Tesar BM, Goldstein DR: Aging impairs IFN regulatory factor 7 up-regulation in plasmacytoid dendritic cells during TLR9 activation. J Immunol 2008, 181:6747-6756.

36. Jing Y, Shaheen E, Drake RR, Chen N, Gravenstein S, Deng Y: Aging is associated with a numerical and functional decline in plasmacytoid dendritic cells, whereas myeloid dendritic cells are relatively unaltered in human peripheral blood. Hum Immunol 2009, 70:777-784.

37. Niessner A, Sato K, Chaikof EL, Colmegna I, Goronzy J, Weyand CM: Pathogen-sensing plasmacytoid dendritic cells stimulate cytotoxic T-cell function in the atherosclerotic plaque through interferon-alpha. Circulation 2006, 114:2482-2489.

38. Deng J, Ma-Krupa W, Gewirtz AT, Younge BR, Goronzy JJ, Weyand CM: Toll-like receptors 4 and 5 induce distinct types of vasculitis. Circ Res 2009, 104:488-495.

39. Goronzy JJ, Fujii H, Weyand CM: Telomeres, immune aging and autoimmunity. Exp Gerontol 2006, 41:246-251.

40. Li M, Fukagawa NK: Age-related changes in redox signaling and VSMC function. Antioxid Redox Signal 2010, 12:641-655.

41. Sawabe M: Vascular aging: from molecular mechanism to clinical significance. Geriatr Gerontol Int 2010, 10 Suppl 1:S213-220.

42. Lee HY, Oh BH: Aging and arterial stiffness. Circ J 2010, 74:2257-2262.

43. Wang M, Monticone RE, Lakatta EG: Arterial aging: a journey into subclinical arterial disease. Curr Opin Nephrol Hypertens 2010, 19:201-207.

44. Gouspillou G, Bourdel-Marchasson I, Rouland R, Calmettes G, Franconi JM, Deschodt-Arsac V, Diolez P: Alteration of mitochondrial oxidative phosphorylation in aged skeletal muscle involves modification of adenine nucleotide translocator. Biochim Biophys Acta 2010, 1797:143-151.

45. Wagner AD, Bjornsson J, Bartley GB, Goronzy JJ, Weyand CM: Interferongamma-producing $T$ cells in giant cell vasculitis represent a minority of tissue-infiltrating cells and are located distant from the site of pathology. Am J Pathol 1996, 148:1925-1933.

46. Mulligan-Kehoe MJ: The vasa vasorum in diseased and nondiseased arteries. Am J Physiol Heart Circ Physiol 2010, 298:H295-305.

doi:10.1186/ar3358

Cite this article as: Mohan SV, et al:: Giant cell arteritis: immune and vascular aging as disease risk factors. Arthritis Research \& Therapy 2011, 13:231. 